Article

\title{
Multiplicative Expression for the Coefficient in Fermionic 3-3 Relation
}

\author{
Igor Korepanov
}

Received: 30 August 2015; Accepted: 15 January 2016; Published: 20 January 2016

Academic Editor: Yuli Rudyak

Moscow Technological University, 20 Stromynka Street, Moscow 107076, Russia; korepanov@mirea.ru

\begin{abstract}
Recently, a family of fermionic relations were discovered corresponding to Pachner move 3-3 and parameterized by complex-valued 2-cocycles, where the weight of a pentachoron (4-simplex) is a Grassmann-Gaussian exponent. Here, the proportionality coefficient between Berezin integrals in the 1.h.s. and r.h.s. of such relations is written in a form multiplicative over simplices.
\end{abstract}

Keywords: four-dimensional TQFT; piecewise-linear TQFT; algebraic realizations of Pachner moves; Grassmann-Gaussian exponents

MSC: 57R56 (Primary); 57Q99; 13P25 (Secondary)

\section{Introduction}

This paper continues a series of papers [1-3]. The reader is referred especially to [3] for definitions and facts that are only briefly mentioned here. In addition, the reader is referred to [4] for a concise exposition of Grassmann-Berezin calculus of anticommuting variables (or to [5] for a more modern and detailed exposition), and to [6] for a pedagogical introduction to Pachner moves.

In [2], a large family was discovered of Grassmann-Gaussian relations corresponding to Pachner move 3-3, with pentachoron (4-simplex) weights depending on a single Grassmann variable attached to each 3-face. In [3], a full parameterization was given for (a Zariski open set of) such relations, in terms of a 2-cocycle given on both 1.h.s. and r.h.s. of the Pachner move. Many questions still remain, however, to be solved before we arrive at a full-fledged four-dimensional topological quantum field theory (TQFT) on piecewise-linear manifolds.

In the present paper, we solve one such question and show that the answer is remarkably nontrivial. It consists in finding the coefficient called 'const' in ([3] formula (53)) (as well as ([2], formula (6)) in a form that would make possible further construction of a manifold invariant. Namely, the coefficient should be represented as a ratio, const $=c_{r} / c_{l}$ (compare relation Equation (2) below), of two expressions belonging to the two sides of the move, and each of these must be multiplicative-have the form of a product over simplices belonging to the corresponding side. This was the case in an earlier paper [1], see formula (1) and Theorem 1 there, also reproduced in ([2] Section 6), although the 3-3 relations in these papers must be regarded as degenerate from the viewpoint of the present paper. This was also the case in ([7], formula (38)) and ([8], formula (12)), where different but similar relations were considered.

\subsection{PL Manifold Invariants and Pachner Moves}

In order to construct invariants of piecewise linear (PL) manifolds, it makes sense to construct algebraic relations corresponding to Pachner moves, see, for instance, ([6], Section 1). Pachner's theorem states that a triangulation of a PL manifold can be transformed into any other triangulation 
using a finite sequence of these moves [9], so there is a hope to pass then from such relations to some quantities characterizing the whole manifold.

More specifically, a popular idea consists in constructing state sum-like invariants. This means the following: a set $X$ of "states", also often called "colors", is introduced for every simplex of some fixed dimension $d$ (for instance, for edges-simplices of dimension $d=1$. Of course, one can also imagine more complicated cases, where simplices of different dimensions are involved). A coloring is then any mapping from the set of all $d$-simplices in triangulation into $X$. In addition, there is a "Boltzmann weight" $W_{u}$, assigned most often to every simplex $u$ of the maximal dimension and depending on the colors of $d$-simplices contained in $u$. The values of $W_{u}$ are supposed to lie in some ring, because the invariant is composed from them using multiplication and addition, as in formula (1) below.

If there is a manifold $M$ with colored boundary $\partial M$ (that is, colors are assigned to all $d$-simplices lying in $\partial M)$, then its weight is defined, typically, as the following sum of products, each corresponding to a coloring of inner $d$-simplices:

$$
W_{M}=\sum_{\substack{\text { over all colorings } \\ \text { of inner } d \text {-simplices }}} \prod_{\text {over all } u} W_{u}
$$

If $M$ is now a result of gluing together two manifolds, $M_{1}$ and $M_{2}$, by means of identifying some (identically triangulated) parts $N_{1} \subset \partial M_{1}$ and $N_{2} \subset \partial M_{2}$ of their boundaries, the weight:

$$
W_{M}=\sum_{\substack{\text { over all colorings } \\ \text { of inner } d \text {-simplices } \\ \text { in } N_{1}=N_{2}}} W_{M_{1}} W_{M_{2}}
$$

has the following obvious property: if we change in any way the triangulation within $M_{1}$ or $/$ and $M_{2}$, but not changing the triangulation of its boundary and not changing its weight, then $W_{M}$ remains the same.

Pachner moves are elementary re-buildings of small clusters of simplices, not changing the topology. In a usual notation, move $m-n$ replaces $m$ simplices of maximal dimension with $n$ simplices. If we have invented the simplex weight such that the cluster weight does not change under all relevant Pachner moves, then Equation (1) gives a manifold invariant.

Such straightforward scheme may work in four dimensions, see, for instance, [10], where all pentachora are assigned the same "constant" weight satisfying the fundamental equation ([10] formula (22)). On the other hand, that "constant" equation may be too restrictive; it may make sense to consider simplex weights depending on parameters having, e.g., some (co)homological meaning.

In the four-dimensional case, the Pachner moves are 3-3, 2-4 and 1-5. The first of them is usually regarded as 'central', and we will be dealing with it in this paper. Here, we describe this move and fix notations for the involved vertices and simplices.

Let there be a cluster of three pentachora (4-simplices) 12345, 12346 and 12356 situated around the 2-face 123. Move 3-3 transforms it into the cluster of three other pentachora, 12456, 13456 and 23456, situated around the 2-face 456. The inner 3-faces (tetrahedra) are 1234, 1235 and 1236 in the 1.h.s., and 1456, 2456 and 3456 in the r.h.s. The boundary of both sides consists of nine tetrahedra.

Note that we have listed in the previous paragraph exactly all simplices in which the 1.h.s. of move 3-3 differs from its r.h.s. The boundary of both sides is, of course, the same, and consists of nine tetrahedra. 


\subsection{Discrete Field Theory}

Our relation corresponding to Pachner move 3-3, or its algebraic realization, that will hopefully lead to a very interesting and new TQFT on PL manifolds, is

$$
\begin{aligned}
& c_{l} \iiint \mathcal{W}_{12345} \mathcal{W}_{12346} \mathcal{W}_{12356} \mathrm{~d} x_{1234} \mathrm{~d} x_{1235} \mathrm{~d} x_{1236} \\
&=c_{r} \iiint \mathcal{W}_{12456} \mathcal{W}_{13456} \mathcal{W}_{23456} \mathrm{~d} x_{1456} \mathrm{~d} x_{2456} \mathrm{~d} x_{3456}
\end{aligned}
$$

This relation has already appeared in (practically) this general form in [1-3]. The integrals in Equation (2) are Berezin integrals [4,5] in Grassmann (anticommuting) variables, and $\mathcal{W}_{i j k l m}$ are Grassmann-Gaussian pentachoron weights explained below; $x_{t}$ are Grassmann variables living on tetrahedra $t$. Thus, the summation over all colorings in Equation (1) has been replaced in Equation (2) by integration in anticommuting variables. The basic reasons behind this are as follows:

- Although it is generally believed that anything "fermionic", like a theory with anticommuting variables, has its "bosonic" parallel-a theory with usual commuting variables, there seems to be no "bosonic" theory as yet with nonconstant relations similar to our relation (2), where, let us recall, the weight parameters are determined by a 2-cocycle. In addition, it was exactly anticommuting variables that appeared naturally in the author's work, although it took quite a while to understand that they are actually hidden behind such formulas as in the short notes [11, 12],

- Grassmann integration is quite a natural operation, it actually leads to finite summation; the basic difference from Equation (1) can be interpreted as "grading": minus signs are inserted in a proper way. Note that, already in 1988, Atiyah in his fundamental paper "Topological quantum field theory" [13] mentioned such a possibility. Here is the exact quotation: "the vector spaces $Z(\Sigma)$ may be mod 2 graded with appropriate signs then inserted", see ([13] p. 181).

As formula (2) contains factors $c_{l}$ and $c_{r}$, it is important, in order to obtain a manifold invariant, to have them expressed in a form multiplicative over simplices. Consider, heuristically, a simplified problem, where a manifold invariant is written in the form analogous to Equation (1), although now it must include some factor $c$ :

$$
c \int \cdots \int \prod_{u} \mathcal{W}_{u} \prod_{\substack{\text { inner } \\ \text { tetrahedra }}} \mathrm{d} x_{t}
$$

and it is very desirable to have an explicit expression for $c$. Now let $c_{l}$ and $c_{r}$ have a multiplicative form, for instance, such as we actually find in this paper, see formulas (60) and (61):

$\prod$ (some values belonging to inner tetrahedra) $\prod$ (some values belonging to pentachora) $\prod$ (some values belonging to inner 2-faces)

Then, one can readily see that invariant Equation (3) works for moves 3-3 with $c$ having the same form (4).

Remark 1. The actual invariant of all Pachner moves will include some more factors in the integrand compared with its "light" version Equation (3), as can be seen, for example, from paper [7], where similar but simpler invariants are considered. The multiplicative form retains, of course, its full importance (compare, for instance, ([7], formula (43))). The work on the extension of our present formulas to all Pachner moves is now in progress.

\subsection{The Results of This Paper and How They Are Explained}

The results are explicit formulas for everything in Equation (2)—that is, Grassmann weights $\mathcal{W}_{i j k l m}$ and coefficients $c_{l}$ and $c_{r}$-in terms of a 2-cocycle $\omega$ introduced in [3] (where not 
everything was calculated explicitly. In particular, only the existence of the mentioned coefficients was shown, see ([3] Theorem 9)). As all formulas are algebraic, the author might have presented just these formulas, saying: and now the validity of Equation (2) can be checked using computer algebra. The formulas look, however, rather intricate, so we follow another way, focusing on the actual author's reasonings.

\section{Explicit Formulas for Matrix Elements}

In this Section, as well as in the next Sections 3 and 4, we work within a single pentachoron $u=12345$. The changes to be made for other $u$ are quite simple and will be explained later.

Convention 1. We denote triangles (2-simplices) by the letter $s$, tetrahedra (3-simplices) by $t$, and pentachora (4-simplices) — by $u$. As for edges (1-simplices), we tend to use the letter $b$ for them, while vertices (0-simplices) are $i, j, k, \ldots$.

Convention 2. We also write the simplices by their vertices, e.g., $s=i j k$ or, as we have written above, $u=12345$. The vertices are thus given by their numbers, and in writing so, we assume by default that the vertices are ordered: $i<j<k$, etc. If, however, we need a triangle whose order of vertices in unknown or unessential, we write $s$ as $\{i j k\}$, as in Lemma 1 below.

\subsection{Edge Operators}

Our Grassmann-Gaussian pentachoron weight is

$$
\mathcal{W}_{u}=\mathcal{W}_{12345}=\exp \left(-\frac{1}{2} x^{\mathrm{T}} F \mathrm{x}\right)
$$

where

$$
\mathrm{x}=\left(\begin{array}{lllll}
x_{2345} & x_{1345} & x_{1245} & x_{1235} & x_{1234}
\end{array}\right)^{\mathrm{T}}
$$

is the column of Grassmann variables corresponding to the 3-faces $t \subset u$, and $F-a 5 \times 5$ antisymmetric matrix.

We are going to recall the construction of matrix $F$ from [3]. Moreover, we will write down some specific explicit expressions for the entries of $F$ that do not appear in [3]. On the other hand, we will skip some details for which the reader is referred to [3].

Our starting point is a 2-cocycle $\omega$ : it takes complex values $\omega_{s}=\omega_{i j k}$ on triangles $s=i j k \subset u$ such that:

$$
\omega_{j k l}-\omega_{i k l}+\omega_{i j l}-\omega_{i j k}=0
$$

Then, there are edge operators $d_{b}$ for the ten edges $b=i j \subset u$ that make the bridge between $\omega$ and matrix $F$. Edge operators have the following properties:

- They belong to the 10-dimensional space of operators:

$$
d=\sum_{t \subset u}\left(\beta_{t} \partial_{t}+\gamma_{t} x_{t}\right)
$$

where $x_{t}$ means left multiplication by this Grassmann variable; $\partial_{t}=\partial / \partial x_{t}$; and $\beta_{t}$ and $\gamma_{t}$ are arbitrary complex coefficients,

- More specifically, the sum (7) for a given $d_{b}$ runs only over such three tetrahedra that $t \supset b$,

- Each of the edge operators annihilates the pentachoron weight:

$$
d_{b} \mathcal{W}_{u}=0
$$


- They are antisymmetric with respect to changing the edge orientation:

$$
d_{i j}=-d_{j i}
$$

- They obey the following linear relations for each vertex $i \in u$ :

$$
\sum_{\substack{j \in u \\ j \neq i}} d_{i j}=0
$$

- In addition, there is one more linear relation:

$$
\sum_{b \subset u} v_{b} d_{b}=0
$$

where $v$ is any 1-cocycle such that $\omega$ makes its coboundary:

$$
\omega=\delta v, \quad \text { i.e., } \quad \omega_{i j k}=v_{j k}-v_{i k}+v_{i j}
$$

- They form a maximal (5-dimensional) isotropic subspace in the (10-dimensional) space of all operators of the form (7), where the scalar product is, by definition, the anticommutator:

$$
\left\langle d^{\prime}, d^{\prime \prime}\right\rangle=\left[d^{\prime}, d^{\prime \prime}\right]_{+}=d^{\prime} d^{\prime \prime}+d^{\prime \prime} d^{\prime}
$$

\subsection{Partial Scalar Products of Edge Operators}

Due to the form (7), we have $t$-components

$$
\left.d_{b}\right|_{t}=\beta_{t} \partial_{t}+\gamma_{t} x_{t}
$$

of edge operators, and the (vanishing) scalar product of two edge operators is a sum over tetrahedra:

$$
0=\left\langle d_{b_{1}}, d_{b_{2}}\right\rangle=\sum_{t \subset u}\left\langle d_{b_{1}}, d_{b_{2}}\right\rangle_{t}
$$

where $\left\langle d_{b_{1}}, d_{b_{2}}\right\rangle_{t}$-we call it the partial scalar product of $d_{b_{1}}$ and $d_{b_{2}}$ with respect to tetrahedron $t$-is by definition the same as $\left\langle\left. d_{b_{1}}\right|_{t},\left.d_{b_{2}}\right|_{t}\right\rangle$.

Lemma 1. Choose a tetrahedron $t \subset u$ and a triangle $\{i j k\} \subset t$ (see Convention 2 for this notation). Then the partial scalar product $\left\langle d_{i j}, d_{i k}\right\rangle_{t}$ remains the same under any permutation of $i, j, k$.

Proof. Let us prove, for instance, that

$$
\left\langle d_{12}, d_{13}\right\rangle_{1234}=\left\langle d_{21}, d_{23}\right\rangle_{1234}
$$

Setting $i=3$ in Equation (9) and taking its $t$-component, we have (keeping in mind also Equation (8)):

$$
-\left.d_{13}\right|_{1234}-\left.d_{23}\right|_{1234}+\left.d_{34}\right|_{1234}=0
$$

We want to take the scalar product of Equation (13) with $d_{12}$. As 1234 is the only tetrahedron common for the edges 12 and 34, and all edge operators are orthogonal to each other, we get:

$$
\left\langle d_{12}, d_{34}\right\rangle_{1234}=\left\langle d_{12}, d_{34}\right\rangle=0
$$

So, the mentioned scalar product, together with Equation (14), gives Equation (12) at once. 
Lemma 2. For a tetrahedron $t \subset u$, construct the expression

$$
\omega_{s}\left\langle d_{b_{1}}, d_{b_{2}}\right\rangle_{t}
$$

Here tetrahedron $t$ is considered as oriented, $s$ is any of its 2-faces with the induced orientation, and $b_{1}, b_{2} \subset s$ are two edges sharing the same initial vertex (thus also oriented). Then, the expression (15) does not depend on a specific choice of $s, b_{1}$ and $b_{2}$, and thus pertains solely to $t$.

Proof. Let us prove, for instance, that

$$
-\omega_{123}\left\langle d_{12}, d_{13}\right\rangle_{1234}=\omega_{124}\left\langle d_{12}, d_{14}\right\rangle_{1234}
$$

(the minus sign accounts for opposite orientations of 123 and 124). A small exercise shows that the following linear relation is a consequence of Equation (10):

$$
-\left.\omega_{123} d_{13}\right|_{1234}-\left.\omega_{124} d_{14}\right|_{1234}+\left.\omega_{234} d_{34}\right|_{1234}=0
$$

Multiplying this scalarly by $d_{12}$ and using once again orthogonality Equation (14), we get Equation (16).

Lemma 3. Expression (15) also remains the same for all tetrahedra t forming the boundary of pentachoron $u$, if these tetrahedra are oriented consistently (as parts of the boundary $\partial u$ ).

Proof. It is enough to consider the situation where $s$ is the common 2-face of two tetrahedra $t, t^{\prime} \subset u$, and show that

$$
\left\langle d_{b_{1}}, d_{b_{2}}\right\rangle_{t}=-\left\langle d_{b_{1}}, d_{b_{2}}\right\rangle_{t^{\prime}}
$$

Indeed, as the orientation of $s$ as part of $\partial t$ is different from its orientation as part of $\partial t^{\prime}$, there are two values $\omega_{s}$ differing in sign, and Equation (17) will yield at once that Equation (15) is the same for $t$ and $t^{\prime}$.

To prove (17), we note that $t$ and $t^{\prime}$ are the only tetrahedra containing both $b_{1}$ and $b_{2}$, so

$$
0=\left\langle d_{b_{1}}, d_{b_{2}}\right\rangle=\left\langle d_{b_{1}}, d_{b_{2}}\right\rangle_{t}+\left\langle d_{b_{1}}, d_{b_{2}}\right\rangle_{t^{\prime}}
$$

Convention 3. We normalize edge operators in such way that quantity (15) becomes unity.

Here is the matrix of scalar products $\left\langle d_{a}, d_{b}\right\rangle_{1234}$ calculated according to Convention 3 . The rows (resp. columns) correspond to edge $a$ (resp. $b$ ) taking values in lexicographic order: 12, 13, 14, 23, 24, 34:

$$
\left(\begin{array}{cccccc}
\omega_{124}^{-1}-\omega_{123}^{-1} & \omega_{123}^{-1} & -\omega_{124}^{-1} & -\omega_{123}^{-1} & \omega_{124}^{-1} & 0 \\
\omega_{123}^{-1} & -\omega_{134}^{-1}-\omega_{123}^{-1} & \omega_{134}^{-1} & \omega_{123}^{-1} & 0 & -\omega_{134}^{-1} \\
-\omega_{124}^{-1} & \omega_{134}^{-1} & \omega_{124}^{-1}-\omega_{134}^{-1} & 0 & -\omega_{124}^{-1} & \omega_{134}^{-1} \\
-\omega_{123}^{-1} & \omega_{123}^{-1} & 0 & \omega_{234}^{-1}-\omega_{123}^{-1} & -\omega_{234}^{-1} & \omega_{234}^{-1} \\
\omega_{124}^{-1} & 0 & -\omega_{124}^{-1} & -\omega_{234}^{-1} & \omega_{124}^{-1}+\omega_{234}^{-1} & -\omega_{234}^{-1} \\
0 & -\omega_{134}^{-1} & \omega_{134}^{-1} & \omega_{234}^{-1} & -\omega_{234}^{-1} & \omega_{234}^{-1}-\omega_{134}^{-1} .
\end{array}\right)
$$

Remark 2. To calculate diagonal elements in matrix (18) is an easy exercise using linear relations similar to Equation (13). 
Remark 3. As for tetrahedron 1235 , we must not only replace ' 4 ' by ' 5 ' in matrix (18), but also change all signs-due to its different orientation! Similarly, analogues of matrix (18) for other tetrahedra can be calculated.

\subsection{Superisotropic Operators and Matrix F}

Superisotropic operators are such operators of the form (7) that annihilate the weight $\mathcal{W}_{u}$ and whose each $t$-component is isotropic, i.e., either $\gamma_{t}=0$ or $\beta_{t}=0$. The rows of matrix $F$ correspond to superisotropic operators in the following sense: every component of the column

$$
\mathrm{p}+F \mathrm{x}
$$

where $x$ is given by Equation (5) and p, similarly, by

$$
p=\left(\begin{array}{lllll}
\partial_{2345} & \partial_{1345} & \partial_{1245} & \partial_{1235} & \partial_{1234}
\end{array}\right)^{\mathrm{T}}
$$

is superisotropic.

We recall ([3] Subsection 4.2) how superisotropic operators proportional to entries of the column (19) are constructed in terms of edge operators. They all are linear combinations written as

$$
g=\sum_{1 \leq i<j \leq 5} \alpha_{i j} d_{i j}, \quad \alpha_{i j} \in \mathbb{C}
$$

First, we choose and fix one of two square roots of each $\omega_{s}$ :

$$
q_{s} \stackrel{\text { def }}{=} \sqrt{\omega_{S}}
$$

Second, we define "initial" $\alpha_{i j}$ as

$$
\alpha_{b}=\prod_{\substack{s \supset b \\ \text { or } s \cap b=\varnothing}} q_{s}
$$

Example 1. As the 2-faces $s \subset 12345$ containing edge 12 are 123, 124 and 125, and the only 2-face not intersecting with 12 is 345 , such "initial" $\alpha_{12}$ is

$$
\alpha_{12}=q_{123} q_{124} q_{125} q_{345}
$$

Finally, the operator proportional to the $i$-th entry in the column (19), and thus corresponding to the tetrahedron $t$ not containing the vertex $i$, is obtained by the following change of signs:

$$
\alpha_{b} \text { remains the same if } b \subset t \text {, else } \alpha_{b} \mapsto-\alpha_{b}
$$

We want to identify the entries in the column (19) with the operators given by Equations (20)-(22). Such identifications are determined to within a renormalization $x_{t} \mapsto x_{t}^{\prime}=\lambda_{t} x_{t}$ of Grassmann variables, implying also $\partial_{i} \mapsto \partial_{i}^{\prime}=\left(1 / \lambda_{i}\right) \partial_{i}$.

Remark 4. This renormalization leads to multiplying matrix $F$ from both sides by the diagonal matrix $\operatorname{diag}\left(\lambda_{2345}^{-1}, \ldots, \lambda_{1234}^{-1}\right)$.

To fix the mentioned arbitrariness, we choose a distinguished edge $a$ in every tetrahedron $t$ and assume that the restriction of $d_{a}$ onto $t$ has a unit coefficient before $\partial_{t}$ :

$$
\left.d_{a}\right|_{t}=\partial_{t}+\gamma x_{t}
$$


As $\left\langle\partial_{t}, x_{t}\right\rangle=1$, this implies

$$
\gamma=\frac{1}{2}\left\langle d_{a}, d_{a}\right\rangle_{t}
$$

Convention 4. In this paper, the distinguished edge $a$ in any tetrahedron $t$ will always be the lexicographically first one, for example, $a=12$ in tetrahedron $t=1234$.

We now denote $g^{(t)}$ the superisotropic operator defined according to Equations (20)-(22). If such operator contains a summand $\gamma x_{t^{\prime}}$, then $\gamma=\left\langle g^{(t)}, d_{a}\right\rangle_{t^{\prime}}$, and if it contains $\beta \partial_{t}$, then $\beta=2 \frac{\left\langle g^{(t)}, d_{a}\right\rangle_{t}}{\left\langle d_{a}, d_{a}\right\rangle_{t}}$. Hence, the matrix element $F_{t t^{\prime}}=\gamma / \beta$ (because the coefficient at $\partial_{t}$ must be set to unity, according to Equation (19)), i.e.,

$$
F_{t t^{\prime}}=\frac{\left\langle g^{(t)}, d_{a}\right\rangle_{t^{\prime}}\left\langle d_{a}, d_{a}\right\rangle_{t}}{2\left\langle g^{(t)}, d_{a}\right\rangle_{t}}
$$

The scalar products are calculated according to Equation (18) and Remark 3.

Example 2. Here is a typical matrix element:

$$
F_{12}=F_{2345,1345}=-\frac{\left(q_{235}^{2}-q_{234}^{2}\right)}{2 q_{134} q_{135} q_{234} q_{235}} \cdot \frac{f_{12}^{(\mathrm{n})}}{f_{12}^{(\mathrm{d})}}
$$

where

$$
\begin{aligned}
f_{12}^{(\mathrm{n})}=q_{124} q_{134} q_{235} q_{345}-q_{125} q_{135} q_{234} q_{345} & +q_{123} q_{135}^{2} q_{245} \\
& -q_{123} q_{134}^{2} q_{245}-q_{124} q_{135} q_{145} q_{235}+q_{125} q_{134} q_{145} q_{234}
\end{aligned}
$$

and

$$
\begin{aligned}
f_{12}^{(\mathrm{d})}=q_{125} q_{134} q_{235} q_{345}-q_{124} q_{135} q_{234} q_{345}- & q_{124} q_{135} q_{235} q_{245} \\
& +q_{125} q_{134} q_{234} q_{245}+q_{123} q_{145} q_{235}^{2}-q_{123} q_{145} q_{234}^{2}
\end{aligned}
$$

\section{Divisors of Matrix Elements}

The central part of the present work consisted in finding a nice description for poles and zeros of matrix elements $F_{t t^{\prime}}$ of the typical form (24). The point is, of course, that the quantities $\omega_{i j k}=q_{i j k}^{2}$ make a cocycle, so there are dependencies

$$
q_{j k l}^{2}-q_{i k l}^{2}+q_{i j l}^{2}-q_{i j k}^{2}=0
$$

for all tetrahedra $i j k l$.

\subsection{Variables $a_{i j}$ and Their Relation to "Initial" $\alpha_{i j}$}

Recall that we are working within one pentachoron 12345 . It has ten 2 -faces, as well as ten edges. This fact, together with the accumulated experience (compare [3] formula (50)), suggests the idea to introduce a 1-chain $a_{i k}$ such that $\omega_{i j k}$ is written as a product of its three values, namely:

$$
\omega_{i j k}=a_{i j} a_{i k} a_{j k}
$$

Given all $\omega_{i j k}$, the $a_{i j}$ are found from the system of equations which become linear after taking logarithms and are easily solved. Interestingly, the result is, up to an overall factor, our old alphas from formula (21):

$$
a_{i j}=p \cdot \alpha_{i j}
$$


where

$$
p=\left(\prod_{\substack{\text { over all 2-faces } i j k \\ \text { of pentachoron } 12345}} \omega_{i j k}\right)^{-1 / 6}
$$

The cocycle relations are now written (instead of Equation (27)) as

$$
a_{k l} a_{j l} a_{j k}-a_{k l} a_{i l} a_{i k}+a_{j l} a_{i l} a_{i j}-a_{j k} a_{i k} a_{i j}=0
$$

Remark 5. We do not permute the indices of $a_{i j}$ in this paper, but if needed, the natural idea is to assume that

$$
a_{i j}=-a_{j i}
$$

\subsection{Matrix Elements in Terms of $a_{i j}$}

Matrix elements $F_{t t^{\prime}}$ can now be calculated in terms of $a_{i j}$. To be exact, here is what we do: set $\alpha_{i j}=a_{i j} / p$ according to Equation (29); the value of $p$ is not of great importance because it will soon cancel out. Then, apply formula (23) with $g^{(t)}$ expressed using Equations (20) and (22); the scalar products are, of course, calculated according to Equation (18), Remark 3, and Equation (28). The following two examples show what we get.

\section{Example 3.}

$$
\begin{aligned}
F_{12}=\frac{a_{25} a_{35}-a_{24} a_{34}}{2 a_{13} a_{14} a_{15} a_{34} a_{35}} & \\
& \cdot \frac{a_{15} a_{34} a_{35}-a_{14} a_{34} a_{35}+a_{14} a_{15} a_{35}-a_{13} a_{15} a_{35}-a_{14} a_{15} a_{34}+a_{13} a_{14} a_{34}}{a_{25} a_{34} a_{35}-a_{24} a_{34} a_{35}-a_{24} a_{25} a_{35}+a_{23} a_{25} a_{35}+a_{24} a_{25} a_{34}-a_{23} a_{24} a_{34}}
\end{aligned}
$$

\section{Example 4.}

$$
\begin{aligned}
F_{21}=-\frac{a_{15} a_{35}-a_{14} a_{34}}{2 a_{23} a_{24} a_{25} a_{34} a_{35}} & \\
& \cdot \frac{a_{25} a_{34} a_{35}-a_{24} a_{34} a_{35}+a_{24} a_{25} a_{35}-a_{23} a_{25} a_{35}-a_{24} a_{25} a_{34}+a_{23} a_{24} a_{34}}{a_{15} a_{34} a_{35}-a_{14} a_{34} a_{35}-a_{14} a_{15} a_{35}+a_{13} a_{15} a_{35}+a_{14} a_{15} a_{34}-a_{13} a_{14} a_{34}}
\end{aligned}
$$

Of course,

$$
F_{12}=-F_{21}
$$

even if it is not immediately obvious from Equations (31) and (32). We will shed some light on this by studying the poles and zeros of these expressions.

\subsection{The Variety of Zeros of the Main Factor in the Denominator of a Matrix Element as Function of} Six Variables

The main factor in the denominator of Equation (31) is

$$
a_{25} a_{34} a_{35}-a_{24} a_{34} a_{35}-a_{24} a_{25} a_{35}+a_{23} a_{25} a_{35}+a_{24} a_{25} a_{34}-a_{23} a_{24} a_{34}
$$

and its pleasing feature is that it depends on the six variables $a_{i j}$ belonging to just one tetrahedron 2345. There is just one dependence between these $a_{i j}$ :

$$
a_{34} a_{35} a_{45}-a_{24} a_{25} a_{45}+a_{23} a_{25} a_{35}-a_{23} a_{24} a_{34}
$$


Lemma 4. The primary decomposition of the affine algebraic variety determined by Equations (31) and (35), and lying in the affine space of six variables $a_{23}, \ldots, a_{45}$, consists of the four irreducible components given by the following primary ideals, which are also already prime:

$$
\begin{gathered}
\left(a_{25}+a_{34}, a_{24}+a_{35}\right) \\
\left(a_{35}, a_{24}\right) \\
\left(a_{34}, a_{25}\right)
\end{gathered}
$$

and

$$
\begin{array}{r}
\left(a_{24} a_{25} a_{34}-a_{24} a_{25} a_{35}-a_{24} a_{34} a_{35}+a_{25} a_{34} a_{35}+a_{24} a_{25} a_{45}-a_{34} a_{35} a_{45},\right. \\
a_{23} a_{25} a_{34}-a_{23} a_{25} a_{35}+a_{23} a_{25} a_{45}-a_{23} a_{34} a_{45}+a_{25} a_{34} a_{45}-a_{34} a_{35} a_{45}, \\
a_{23} a_{24} a_{35}-a_{23} a_{25} a_{35}-a_{23} a_{24} a_{45}+a_{24} a_{25} a_{45}+a_{23} a_{35} a_{45}-a_{24} a_{35} a_{45}, \\
\left.a_{23} a_{24} a_{34}-a_{23} a_{25} a_{35}+a_{24} a_{25} a_{45}-a_{34} a_{35} a_{45}\right)
\end{array}
$$

Proof. Direct calculation using Singular computer algebra system.

Remark 6. The reader may notice that some more computer calculations of primary decompositions might have been helpful in the process of doing this work. They are, however, more difficult, and the calculation in Lemma 4 is typical of what the available computer capabilities allowed us to do-and this proved to be enough for achieving the goal of this work.

While there is no problem understanding the structure of components (36)-(38), the component (39) deserves the following lemma.

Lemma 5. The affine algebraic variety determined by the ideal (39), and lying in the space of six variables $a_{23}, \ldots, a_{45}$, admits the following trigonometric parameterization:

$$
a_{i j}=c \cdot \tan \left(x_{i}-x_{j}\right)
$$

It is thus rational, because parameterization Equation (40) becomes rational if re-written in terms of $c$ and tangents of three independent differences of $x_{i}$.

Proof. It is enough to substitute Equation (40) into each of the four generators of the prime ideal (39), and check that all of them vanish.

\subsection{Divisor of a Matrix Element: Almost Full Description, Excluding Only Subvarieties $a_{i j}=0$}

We consider the affine algebraic variety $\mathcal{M}$ in the space of ten variables $a_{12}, \ldots, a_{45}$, defined by the relations (30) for all tetrahedra $i j k l \subset u=12345$. Then, we consider its Zariski open subspace $\mathcal{M}^{\prime}$ defined as follows:

$$
\mathcal{M}^{\prime}=\left(\mathcal{M} \text { minus all subvarieties where some } a_{i j}=0\right)
$$

Remark 7. The goal of this paper consists in finding the expressions (60) and (61) below, for example, by guess. It looks hardly possible to guess these expressions if based on nothing, while studying divisors on $\mathcal{M}^{\prime}$ has proved to provide a good basis for the correct guess, as we will see. So, we content ourself with $\mathcal{M}^{\prime}$. Nevertheless, studying divisors on the whole $\mathcal{M}$ might be also of interest, because, for instance, divisors (37) and (38) lie exactly in $\mathcal{M} \backslash \mathcal{M}^{\prime}$.

In $\mathcal{M}^{\prime}$, we introduce the following subvarieties of codimension 1 , denoted as $D$ with indices because we think of them as Weil divisors: 
- $D_{u}$ : this is the subvariety given by the old formulas (40), but now ten of them: $1 \leq i \leq j \leq 5$,

- $\left(D_{u}\right)_{K}$ : choose now subset $K \subset\{1,2,3,4,5\}$, and define $\left(D_{u}\right)_{K}$ by the same formulas (40) except that we change the signs of those $a_{i j}$ whose exactly one subscript $i$ or $j$ is in $K$. We write also $\left(D_{u}\right)_{1},\left(D_{u}\right)_{12}$, etc. instead of $\left(D_{u}\right)_{\{1\}},\left(D_{u}\right)_{\{1,2\}}$, etc.,

- $D_{t}^{-}$: for a tetrahedron $t=i j k l \subset u$, let $b=i j$ be the distinguished edge. Then $D_{t}^{-}$is given by the following equations (compare to (36)):

$$
D_{t}^{-}: \quad\left\{\begin{array}{l}
a_{i k}=-a_{j l} \\
a_{j k}=-a_{i l}
\end{array}\right.
$$

- $D_{t}^{+}$, similarly:

$$
D_{t}^{+}:\left\{\begin{array}{l}
a_{i k}=a_{j l} \\
a_{j k}=a_{i l}
\end{array}\right.
$$

Lemma 6. For a tetrahedron $t=i j k l$ and its distinguished edge $i j$, the sum $D_{t}^{-}+D_{t}^{+}$gives, on $\mathcal{M}^{\prime}$, exactly the zero divisor of $\frac{\sigma_{t}}{a_{i j}}=a_{j k} a_{i k}-a_{j l} a_{i l}$ (compare with the first factor in the numerator of either Equation (31) or (32)!), where we denoted

$$
\sigma_{t}=\omega_{i j k}-\omega_{i j l}
$$

Proof. Due to the cocycle relation (6),

$$
\left(\sigma_{t}=0 \text { on } \mathcal{M}^{\prime}\right) \Leftrightarrow\left(a_{i k} a_{j k}-a_{i l} a_{j l}=0 \text { and } a_{i k} a_{i l}-a_{j k} a_{j l}=0\right)
$$

and the r.h.s. clearly gives Equation (42) or Equation (43).

Lemma 7. For every triangle $s=i j k$, introduce the quantity

$$
\psi_{s}=a_{j k}-a_{i k}+a_{i j}
$$

Trigonometric parameterization (40) specifies, on the subset where all $a_{i j} \neq 0$, the variety that can be given by the system of equations of the following form:

$$
\frac{\psi_{s}}{\omega_{s}} \text { is the same for all } s
$$

This applies to the case where the indices in Equation (40) take four (like in Lemma 5) as well as five values (or, in fact, any number of them).

Proof. Direct calculation.

Theorem 8. (i) The pole divisor of matrix element (31), restricted to $\mathcal{M}^{\prime}$, is $D_{u}$.

(ii) The zero divisor of (31), restricted to $\mathcal{M}^{\prime}$, is $\left(D_{u}\right)_{12}+D_{2345}^{+}+D_{1345}^{+}$(the last two are defined in (43)).

Proof. First, note that the component (36) of the divisor of function (34) cancels out with the first factor in the numerator of (31), that is,

$$
a_{25} a_{35}-a_{24} a_{34}
$$

and what remains of the zero divisor of (45) after this canceling is $D_{2345}^{+}$, according to Lemma 6 .

For item (i), this means that, on the pole variety of (31), all the expressions $\psi_{s} / \omega_{s}$ are the same for $s \subset$ 2345. In addition, analyzing (32) similarly (and taking into account (33)), we arrive at the conclusion that the same are also $\psi_{s} / \omega_{s}$ for $s \subset 1345$. It is not hard to deduce now (through a small 
calculation) that $\psi_{s} / \omega_{s}$ are the same for the whole pentachoron 12345 , including $s=123,124$ and 125 . So, item (i) is proved.

For item (ii), we first notice that the main factor in the numerator of $F_{12}$ (resp. $F_{21}$ ) is the same (up to an overall sign) as the main factor in the denominator of $F_{21}$ (resp. $F_{12}$ ) except that the sign is changed of all $a_{i j}$ with $i=1$ (resp. $i=2$ ). For $F_{12}$, this means that $D_{1345}^{+}$appears as a component of zero divisor, in analogy with (36), while the first paragraph of this proof means that $D_{2345}^{+}$is also there. The rest, namely $\left(D_{u}\right)_{12}$, appears in full analogy with $D_{u}$ in the previous paragraph. So, item (ii) is also proved.

Remark 8. Theorem 8 speaks about a specific matrix element and divisors. It applies, however, to all similar objects, with obvious changes.

\section{Function $\varphi_{12345}$}

On our subvariety $\mathcal{M}^{\prime} \subset \mathcal{M}(41)$, we can express all $a_{i j}$ in terms of $q_{i j k}$ according to (29), where the factor $p$ never vanishes and can be ignored as long as we are considering the zero or pole varieties of expressions homogeneous in variables $a_{i j}$.

Remark 9. All functions of $a_{i j}$ or $q_{i j k}$ in this paper are homogeneous.

Remark 10. In addition, the fact that $p$ is multivalued makes no obstacle in our way.

Convention 5. We will denote, taking some liberty, that part of the variety of variables $q_{i j k}, 1 \leq i<$ $j<k \leq 5$, where all $q_{i j k} \neq 0$, by the same letter $\mathcal{M}^{\prime}$ as the similar variety in variables $a_{i j}$. It is implied of course that the $q_{i j k}$ obey the cocycle relations (27). In addition, we will use the old notations like $\left(D_{u}\right)_{K}$ and $D_{t}^{ \pm}$for codimension one subvarieties in $\mathcal{M}^{\prime}$ that are like in Section 3.4 except that we made the substitution (29) in their defining equations.

For every 3-face $t$ of pentachoron 12345, we define expression $f^{(t)}$ as the biggest factor in the denominator of type (24), namely:

$$
\begin{gathered}
f^{(2345)}=q_{125} q_{134} q_{235} q_{345}-q_{124} q_{135} q_{234} q_{345}-q_{124} q_{135} q_{235} q_{245} \\
+q_{125} q_{134} q_{234} q_{245}+q_{123} q_{145} q_{235}^{2}-q_{123} q_{145} q_{234}^{2} \\
f^{(1345)}=q_{124} q_{134} q_{235} q_{345}-q_{125} q_{135} q_{234} q_{345}-q_{123} q_{135}^{2} q_{245} \\
+q_{123} q_{134}^{2} q_{245}+q_{124} q_{135} q_{145} q_{235}-q_{125} q_{134} q_{145} q_{234} \\
f^{(1245)}=q_{123} q_{125}^{2} q_{345}-q_{123} q_{124}^{2} q_{345}-q_{124} q_{134} q_{235} q_{245} \\
+q_{125} q_{135} q_{234} q_{245}-q_{125} q_{134} q_{145} q_{235}+q_{124} q_{135} q_{145} q_{234} \\
f^{(1235)}=q_{124} q_{125}^{2} q_{345}-q_{123}^{2} q_{124} q_{345}-q_{123} q_{134} q_{235} q_{245} \\
-q_{125} q_{134} q_{135} q_{245}+q_{125} q_{145} q_{234} q_{235}+q_{123} q_{135} q_{145} q_{234} \\
f^{(1234)}=q_{124}^{2} q_{125} q_{345}-q_{123}^{2} q_{125} q_{345}-q_{123} q_{135} q_{234} q_{245} \\
-q_{124} q_{134} q_{135} q_{245}+q_{124} q_{145} q_{234} q_{235}+q_{123} q_{134} q_{145} q_{235}
\end{gathered}
$$

Remark 11. The overall sign of any of expressions (46)-(50) is not now of big importance.

In addition, for every subset $K \subset\{i, j, k, l\}$ consider function $f_{K}^{(t)}$ made from (46)-(50) as follows: change the signs at those $q_{i j k}$ having an odd number of subscripts (one or all three of $i, j$ and $k$ ) is in $K$. In the next Lemma 9 we go through the 3-faces of 12345 in their natural order, and write down the zero divisors of some interesting functions on $\mathcal{M}^{\prime}$. 
Lemma 9. - $f^{(2345)}$ has zero divisor $D_{u}+\left(D_{u}\right)_{1}+D_{2345}^{-}$,

- $f_{1}^{(1345)}$ has zero divisor $\left(D_{u}\right)_{1}+\left(D_{u}\right)_{12}+D_{1345^{\prime}}^{+}$

- $f_{12}^{(1245)}$ has zero divisor $\left(D_{u}\right)_{12}+\left(D_{u}\right)_{123}+D_{1245^{\prime}}^{-}$

- $f_{123}^{(1235)}$ has zero divisor $\left(D_{u}\right)_{123}+\left(D_{u}\right)_{1234}+D_{1235}^{+}$,

- $f_{1234}^{(1234)}=f^{(1234)}$ has zero divisor $\left(D_{u}\right)_{1234}+\left(D_{u}\right)_{12345}+D_{1234}^{-}=\left(D_{u}\right)_{5}+D_{u}+D_{1234}^{-}$.

Thus, on $\mathcal{M}^{\prime}$, the function

$$
\frac{f^{(2345)} f_{12}^{(1245)} f^{(1234)}}{f_{1}^{(1345)} f_{123}^{(1235)}}
$$

has the divisor (zeros with sign plus, poles with sign minus)

$$
2 D_{u}+D_{2345}^{-}-D_{1345}^{+}+D_{1245}^{-}-D_{1235}^{+}+D_{1234}^{-}
$$

Proof. The formulas for divisors of the first five functions make a simple variations on the theme of Lemma 4, where, of course, Convention 5 must be also taken into account. Then, (52) follows by adding/subtracting relevant divisors.

Motivated by Lemma 6, we divide the expression (51) by

$$
\sigma_{2345} \sigma_{1245} \sigma_{1234}=\left(\omega_{234}-\omega_{235}\right)\left(\omega_{124}-\omega_{125}\right)\left(\omega_{123}-\omega_{124}\right)
$$

Theorem 10. The divisor of the so obtained expression

$$
\varphi_{12345}=\frac{f^{(2345)} f_{12}^{(1245)} f^{(1234)}}{\sigma_{2345} \sigma_{1245} \sigma_{1234} f_{1}^{(1345)} f_{123}^{(1235)}}
$$

considered as a function on $\mathcal{M}^{\prime}$, is

$$
2 D_{u}-\sum_{t \subset u} D_{t}^{+}
$$

Proof. This follows from (52) and Lemma 6.

Divisor (54) is thus symmetric under all permutations of 3-faces $t$ of pentachoron $u$. This suggests the idea that function $\varphi_{12345}$ may also remain the same, to within a possible sign change, under any permutation of vertices $1, \ldots, 5$. Basically, this idea turns out to be right, but we do not go into details here; these details include, in particular, choosing the right signs of $q_{i j k}=\sqrt{\omega_{i j k}}$ after we have changed the sign of $\omega_{i j k}$ itself due to permuting its indices.

\section{The Poles and Zeros of the Coefficient in 3-3 Relation, and Its Explicit Form}

We now pass from the single pentachoron 12345 to Pachner move 3-3, where six pentachora are involved.

The 1.h.s. and r.h.s. of move 3-3 are triangulated manifolds with boundary. We can orient the pentachora in both these manifolds consistently, and also so that these orientations induce the same orientation on the boundary $\partial$ (1.h.s.) $=\partial$ (r.h.s.). For one such orientation (of two), the signs in the following table show whether this consistent orientation of a pentachoron coincides with the orientation given by the natural order of its vertices:

$$
\begin{array}{cccccc}
\multicolumn{3}{c}{\text { left-hand side }} & \multicolumn{4}{c}{\text { right-hand side }} \\
12345 & 12346 & 12356 & 12456 & 13456 & 23456 \\
+ & - & + & + & - & +
\end{array}
$$

We do now all calculations in terms of variables $q_{i j k}$ and not $a_{i j}$. This is due to the following important remark. 
Remark 12. Variables $a_{i j}$ depend on a pentachoron (i.e., two $a_{i j}$ for the same edge $i j$, but calculated within two different pentachora containing this edge, are different), while $q_{i j k}$ do not.

\subsection{Matrix Elements for All Six Pentachora Involved in Move 3-3}

In Section 2, we explained how to calculate matrix $F$ elements for pentachoron 12345 . For any pentachoron $i j k l m$ (recall that $i<\cdots<m$, according to Convention 2), the obvious substitution $1 \mapsto i, \ldots, 5 \mapsto m$ must be made. Besides this, the sign of matrix element must be changed for the pentachora marked with minus sign in table (55), as we are going to explain in (the proof of) Lemma 11, where we study the way how our normalization of edge operators, given by Convention 3 , propagates from one pentachoron to another.

Lemma 11. Expression (15) can be normalized to unity for a whole oriented triangulated manifold.

Proof. Let tetrahedron $t$ be the common 3-face of two pentachora, $t=u_{1} \cap u_{2}$. Let $a \subset t$ be its edge, and $d_{a}^{\left(u_{1}\right)}$ and $d_{a}^{\left(u_{1}\right)}$ - the corresponding edge operators in our two pentachora. Then,

$$
\begin{aligned}
\text { if } & \left.d_{a}^{\left(u_{1}\right)}\right|_{t}=\beta_{t} \partial_{t}+\gamma_{t} x_{t} \\
\text { then } & \left.d_{a}^{\left(u_{2}\right)}\right|_{t}=\beta_{t} \partial_{t}-\gamma_{t} x_{t}
\end{aligned}
$$

see ([3] formulas (58)).

We see now that, on passing to a neighboring pentachoron, first, the orientation of $t$ changes (and this affects the orientation of $s$ in(15)!), and second-partial scalar products of edge operators also change their signs because of (56). Hence, the quantity (15) remains the same, as before in Lemmas 1, 2 and 3. This means that it pertains to the whole triangulated manifold, if it is orientable and connected. Hence, we can normalize all edge operators globally so that quantity (15) stays always equal to unity.

In addition, it is clear from (23) that, indeed, changing the sign of partial scalar products implies changing the sign of matrix elements.

\subsection{Components in the l.h.s. and r.h.s., Their Poles and Zeros}

Triple integrals in (2) are polynomials in Grassmann variables, and their coefficients are proportional. A typical coefficient, namely one at $x_{1245}$ (the Grassmann variable corresponding to tetrahedron 1245), is

$$
L=F_{1234,1236} F_{1235,1245}-F_{1235,1236} F_{1234,1245}
$$

in the 1.h.s., and

$$
R=F_{1456,3456} F_{2456,1245}-F_{2456,3456} F_{1456,1245}
$$

in the r.h.s.

Remark 13. Two tetrahedra in the subscripts of a matrix element in (57) or (58) clearly determine the relevant pentachoron.

Our goal is now to guess the form of $c_{l}$ and $c_{r}$. As we can then check the correctness of our guess with a direct computer calculation, informal reasoning will be quite enough for us at this moment.

So, we analyze poles and zeros of $L, R$, and other similar Grassmann polynomial coefficients, in order to invent such $c_{l}$ and $c_{r}$ that will compensate these poles and zeros. First, we do so assuming that no one of values $q_{i j k}$ vanish, that is, within the 'global analogue' of set $\mathcal{M}^{\prime}$ (41). The poles of at least one component in the l.h.s. are relevant, while the zeros must be common for all components; similarly for r.h.s. We see this way that the poles are situated on divisors $D_{u}$ (see Section 3.4) for all 
pentachora in the relevant side of Pachner move, while the zeros are situated on divisors $D_{t}^{+}$of all inner tetrahedra, again in the relevant side of Pachner move.

\subsection{Fitting the Divisors, and the Formulas for $c_{l}$ and $c_{r}$}

The above analysis of poles and zeros of triple Berezin integrals in (2), when confronted with the divisor (54) of function $\varphi_{12345}$, suggests that square roots of such functions may be the key ingredient of our $c_{l}$ and $c_{r}$. So, we introduce, in analogy with $\varphi_{12345}$, quantities $\varphi_{u}$ for each pentachoron $u$ (simply making relevant subscript substitutions).

Now, we look at what may happen where some $q_{i j k}$ do vanish. Motivated by the products of $q_{i j k}$ factored out in the denominators of expressions like (24), we introduce the quantities

$$
\varrho_{t}=q_{i j k} q_{i j l}
$$

for tetrahedra $t=i j k l$. These $\varrho_{t}$ are expected to compensate the poles appearing where the mentioned denominators vanish.

Remark 14. Note that 2-faces $i j k$ and $i j l$ in (59) both contain the distinguished edge $i j \subset t$, see Section 2.3.

What remains is a bit more guessing, trying, scrutinizing formulas, and we arrive at the following theorem:

Theorem 12. For the 3-3 relation (2) to hold, it is enough to set its left-hand-side coefficient to

$$
c_{l}=\varrho_{1234} \varrho_{1235} \varrho_{1236} \sqrt{\varphi_{12345}} \sqrt{\varphi_{12346}} \sqrt{\varphi_{12356}} / q_{123}
$$

and its right-hand-side coefficient to

$$
c_{r}=\varrho_{1456} \varrho_{2456} \varrho_{3456} \sqrt{\varphi_{12456}} \sqrt{\varphi_{13456}} \sqrt{\varphi_{23456}} / q_{456}
$$

Proof. As the proportionality of Grassmann polynomials in the two sides in (2) has been already established in [3], it is enough to compare the coefficients in the 1.h.s. and r.h.s. at any one specific monomial. For instance, it is enough to show that

$$
c_{l} L=c_{r} R
$$

where $L$ and $R$ are given by (57) and (58). Now, we note that both sides in (62) are functions of the values $\omega_{i j k}$ of our cocycle $\omega$, obtained using arithmetic operations (addition, subtraction, multiplication and division) and also taking square roots-but nothing more involved. The values $\omega_{i j k}$ are not independent, but we can pass to independent variables $v_{i j}$, see (11). Of the fifteen $v_{i j}, 1 \leq i<j \leq 6$, five can be set to zero without loss of generality, e.g., all those with $i=1$.

Expressing everything in (62) in terms of the ten remaining $v_{i j}$ and using some computer algebra, we see that (62) indeed holds.

A small miracle in formulas (60) and (61) is the denominators $q_{123}$ and $q_{456}$, appearing because exactly such a value factors out in a non-obvious way in the numerator of $L$ or $R$ during the reduction to common denominator.

Acknowledgments: I thank Evgeniy Martyushev for his interest in this work. He made also some interesting calculations; although they are not used directly in this paper, they showed me some beautiful things and added thus to my inspiration. I am also grateful to creators and maintainers of Maxima and Singular computer algebra systems for their great work. 
Conflicts of Interest: The author declares no conflict of interest.

\section{References}

1. Korepanov, I.G. Special 2-cocycles and 3-3 Pachner move relations in Grassmann algebra. 2013, arXiv:1301.5581.

2. Korepanov, I.G.; Sadykov, N.M. Parameterizing the simplest Grassmann-Gaussian relations for Pachner move 3-3. SIGMA 2013, 9, 53, arXiv:1305.3246.

3. Korepanov, I.G. Two-cocycles give a full nonlinear parameterization of the simplest 3-3 relation. Lett. Math. Phys. 2014, 104:10, 1235-1261.

4. Berezin, F.A. The Method of Second Quantization; Academic Press: New York, NY, USA, 1966.

5. Berezin, F.A. Introduction to Superanalysis. In Mathematical Physics and Applied Mathematics; Kirillov, A.A., Ed.; D. Reidel: Dordrecht, The Netherlands, 1987; p. 424.

6. Lickorish, W.B.R. Simplicial moves on complexes and manifolds. Geom. Topol. Monogr. 1999, 2, $299-320$.

7. Korepanov, I.G. Relations in Grassmann algebra corresponding to three- and four-dimensional Pachner moves. SIGMA 2011, 7, 117, arXiv:1105.0782.

8. Korepanov, I.G. Deformation of a $3 \rightarrow 3$ Pachner move relation capturing exotic second homologies. 2012, arXiv:1201.4762.

9. Pachner, U. PL homeomorphic manifolds are equivalent by elementary shellings. Eur. J. Combinatorics 1991, 12, 129-145.

10. Kashaev, R.M. On realizations of Pachner moves in 4D. 2015, arXiv:1504.01979.

11. Korepanov, I.G. A formula with volumes of five tetrahedra and discrete curvature. 2000, arXiv:nlin/0003001.

12. Korepanov, I.G. A formula with hypervolumes of six 4-simplices and two discrete curvatures. 2000, arXiv:nlin/0003024.

13. Atiyah, M.F. Topological quantum field theory. Publ. Mathématiques de l'I.H.É.S. 1988, 68, 175-186.

(c) 2016 by the author; licensee MDPI, Basel, Switzerland. This article is an open access article distributed under the terms and conditions of the Creative Commons by Attribution (CC-BY) license (http://creativecommons.org/licenses/by/4.0/). 